\title{
Dynamics of exciton dissociation in donor- acceptor polymer heterojunctions
}

\author{
Zhen Sun and Sven Stafström
}

\section{Linköping University Post Print}

\section{Tweet}

N.B.: When citing this work, cite the original article.

Original Publication:

Zhen Sun and Sven Stafström, Dynamics of exciton dissociation in donor-acceptor polymer heterojunctions, 2013, Journal of Chemical Physics, (138), 16.

http://dx.doi.org/10.1063/1.4802764

Copyright: American Institute of Physics (AIP) http://www.aip.org/

Postprint available at: Linköping University Electronic Press

http://urn.kb.se/resolve?urn=urn:nbn:se:liu:diva-94327 


\title{
Dynamics of exciton dissociation in donor-acceptor polymer heterojunctions
}

\author{
Zhen Sun ${ }^{\text {a) }}$ and Sven Stafström ${ }^{\text {b) }}$ \\ Department of Physics, Chemistry, and Biology, Linköping University, SE-58183 Linköping, Sweden
}

(Received 3 December 2012; accepted 9 April 2013; published online 29 April 2013)

\begin{abstract}
Exciton dissociation in a donor-accepter polymer heterojunction has been simulated using a nonadiabatic molecular dynamics approach, which allows for the coupled evolution of the nuclear degrees of freedom and the electronic degrees of freedom described by multiconfigurational electronic wavefunctions. The simulations reveal important details of the charge separation process: the exciton in the donor polymer first dissociates into a "hot" charge transfer state, which is best described as a polaron pair. The polaron pair can be separated into free polaron charge carriers if a sufficiently strong external electric field is applied. We have also studied the effects of inter-chain interaction, temperature, and the external electric field strength. Increasing inter-chain interactions makes it easier for the exciton to dissociate into a polaron pair state, but more difficult for the polaron pair to dissociate into free charge carriers. Higher temperature and higher electric field strength both favor exciton dissociation as well as the formation of free charge carriers. (c) 2013 AIP Publishing LLC. [http://dx.doi.org/10.1063/1.4802764]
\end{abstract}

\section{INTRODUCTION}

Organic solar cells are currently of broad interest due to their potential use in low-cost, light-weight, large-area photovoltaic solar cells. It is a key challenge in any solar cell application to enhance the power conversion efficiency. Following the development of the bulk heterojunction structure, ${ }^{1-3}$ further improvements in terms of materials with increased light absorbing properties and so called tandem cells, ${ }^{4,5}$ the organic solar cells have reached power conversion efficiencies of about $10 \% .^{6,7}$ However, compared to inorganic solar cell, the efficiencies of organic solar cells need further improvement.

Since the primary photoexcited species in organic solar cells are singlet excitons, rather than free charge carriers, ${ }^{8,9}$ the power conversion efficiency relies on efficient exciton dissociation into free charge carriers. The Coulomb interaction together with the electron-lattice interactions causes a large binding energy for the exciton, typically of the order of $0.5 \mathrm{eV},{ }^{10,11}$ which is significantly larger than the thermal energy $k_{B} T$. As a result, a simple single-layer device based on the pristine polymer and two electrodes of different work functions has poor power conversion and charge collection efficiencies. $^{12}$

Over the past few decades, heterojunctions composed of electron-donating semiconducting polymers and electronaccepting fullerides have been widely used to make high efficient photovoltaic cells (for reviews, see Refs. 13-15). The heterojunction creates a band offset at the interface between the two materials, which helps to overcome the large exciton binding energy and enabling the electron transfer from the donor polymer to the acceptor polymer. ${ }^{16-18}$ Thus, to im-

a)Electronic mail: zhesu@ifm.liu.se

b) Electronic mail: svens@ifm.liu.se prove the probability of exciton dissociation, a large band offset should be preferred. However, a large band offset has the disadvantage to reduce the available open-circuit voltage. ${ }^{19}$ Consequently, there is an optimal value for the band offset, which maximizes the power conversion efficiency.

The process from the absorption of photons to the extraction of free charge carriers is a multi-step process: (i) the absorption of a photon, usually by the donor, generates an exciton, (ii) the exciton diffuses to the donor/acceptor (D/A) interface and dissociates into a charge transfer (CT) state, a Coulombically bound electron-hole pair located on adjacent donor and acceptor polymers with the binding energy about $0.1-0.5 \mathrm{eV},{ }^{20,21}$ and (iii) separation of charges in the CT state into free charge carriers, a process which has to be assisted by the cell's internal electric field. A detailed understanding of this multistep process is a key to further enhance the efficiency of organic solar cell. . $^{14,22,23}$

In this paper, we focus on steps (ii) and (iii), that is, the exciton dissociation into CT state and the following charge separation state at the D/A interface. The D-A polymer heterojunction is in our model system composed of two parallel chains in the configuration D-A, where D represents the polymer with lower electron affinity, and A represents the polymer with higher electron affinity. Initially, we create a singlet exciton in the D-chain, then we smoothly turn on the inter-chain interactions to mimic the process of the exciton moving towards the D-A polymer heterojunction. To get some insight into the separation of the polaron pair into free polarons in a direction perpendicular to the heterojunction, we additionally carried out simulations of the same process in a D-D-AA polymer heterojunction which is composed of four parallel chains in the configuration D-D-A-A. For this configuration, the exciton is initially located on the inner D-chain. By simulating this process using nonadiabatic molecular dynamics method, we can present a detailed description of the exciton 
dissociation at the atomistic level and with a time resolution at the sub-femtosecond timescale. The effects of factors such as inter-chain coupling, temperature, and electric field are also investigated.

\section{MODEL AND METHOD}

The total Hamiltonian of our model system can be expressed as

$$
\hat{H}=\hat{H}_{\text {elec }}+\hat{H}_{\text {latt }} .
$$

The first term in Eq. (1) is the Hamiltonian of the electronic part of the system, which is expressed as

$$
\hat{H}_{\text {elec }}=\hat{H}_{\text {intra }}+\hat{H}_{\text {inter }},
$$

where $\hat{H}_{\text {intra }}$ describes intra-chain interactions, which has the form

$$
\hat{H}_{\text {intra }}=\hat{H}_{e l}+\hat{H}_{e e}+\hat{H}_{E} .
$$

In the above expression, the first term represents the interactions experienced by the single electron:

$$
\hat{H}_{e l}=\sum_{i, s} \Delta_{i} \hat{c}_{i, s}^{\dagger} \hat{c}_{i, s}-\sum_{i, s} t_{i, i+1}\left(\hat{c}_{i, s}^{\dagger} \hat{c}_{i+1, s}+\hat{c}_{i+1, s}^{\dagger} \hat{c}_{i, s}\right)
$$

where $i$ runs over the sites of all the donor and acceptor chains (a simplified notation is used without a chain index on $i$ ). $\Delta_{i}$ denotes the on-site energy of site $i$. To mimic the D-A (D-DA-A) polymer heterojunction, we set different values of the on-site energy on the D-chain and A-chain, $\Delta_{i}=\Delta E$ for all sites of the D-chain and $\Delta_{i}=0$ for all sites of A-chain. $\hat{c}_{i, s}^{\dagger}$ and $\hat{c}_{i, s}$ are the creation and annihilation operators for a $\pi$ electron with spin $s$ at site $i$ on one of the chains,

$$
t_{i, i+1}=t_{0}-\alpha\left(u_{i+1}-u_{i}\right)+(-1)^{i} t_{e}
$$

is the intra-chain nearest-neighbor transfer integral, with $t_{0}$ denoting the transfer integral for zero displacement, $\alpha$ the electron-phonon coupling constant, $u_{i}$ the displacement on the $i$ th site from equidistant position between neighboring carbon atoms, and $t_{e}$ the symmetry-breaking term, introduced to lift the ground-state degeneracy for non-degenerate polymers.

The second contribution in Eq. (3) describes the intrachain electron-electron interactions:

$$
\begin{aligned}
\hat{H}_{e e}= & U \sum_{i}\left(\hat{n}_{i \uparrow}-\frac{1}{2}\right)\left(\hat{n}_{i \downarrow}-\frac{1}{2}\right) \\
& +\frac{1}{2} \sum_{i, j \neq i} V_{i j}\left(\hat{n}_{i}-1\right)\left(\hat{n}_{j}-1\right),
\end{aligned}
$$

where $U$ denotes the screened on-site Coulomb interactions, $\hat{n}_{i, s}=\hat{c}_{i, s}^{\dagger} \hat{c}_{i, s}, \hat{n}_{i}=\sum_{s} \hat{c}_{i, s}^{\dagger} \hat{c}_{i, s}$, and $V_{i j}$ is the Ohno potential defined as ${ }^{24}$

$$
V_{i j}=\frac{U}{\sqrt{1+\left(\beta r_{i j} / r_{0}\right)^{2}}},
$$

where $i$ and $j$ run over the sites of the same chain. The Ohno potential describes the long-range Coulomb interactions, where $r_{i j}$ denotes the distance between sites $i$ and $j, r_{0}$ the average bond length, and $\beta$ the screening factor.
The last contribution in Eq. (3) represents the external electric field, $E(t)$,

$$
\hat{H}_{E}=|e| E(t) \sum_{i, s}\left[(i) a+u_{i}\right]\left(\hat{n}_{i, s}-\frac{1}{2}\right),
$$

where $e$ denotes the electronic charge. In our simulations, $E(t)$ is constant after a smooth turn on.

In Eq. (2), $\hat{H}_{\text {inter }}$ describes the inter-chain interactions, which has the form of

$$
\begin{aligned}
\hat{H}_{i n t e r}= & \sum_{\langle i, j\rangle, s}-t_{i, j}\left(\hat{c}_{i, s}^{\dagger} \hat{c}_{j, s}+\hat{c}_{j, s}^{\dagger} \hat{c}_{i, s}\right) \\
& +\frac{1}{2} \sum_{i, j} V_{i j}\left(\hat{n}_{i}-1\right)\left(\hat{n}_{j}-1\right),
\end{aligned}
$$

where $i$ and $j$ run over the sites of neighbor chains, respectively. The symbol of $\sum_{\langle i, j\rangle}$ means that the sum is restricted to pairs of nearest and next nearest neighboring sites in the opposite chains, and $t_{i, j}$ denotes the inter-chain hopping integral. This quantity is calculated as a function of the inter-chain distance, $d$, using

$$
t_{i, j}= \begin{cases}\frac{t_{0}}{10} \exp \left(1-\frac{d}{5}\right) & \text { nearest hopping, } \\ \frac{t_{0}}{10} \exp \left(1-\frac{\sqrt{d^{2}+r_{0}^{2}}}{5}\right) & \text { next nearest hopping. }\end{cases}
$$

$V_{i, j}$ describes the inter-chain electron-electron interactions.

The last term in Eq. (1) is the Hamiltonian of the lattice backbone, which is treated classically:

$$
\hat{H}_{l a t t}=\frac{K}{2} \sum_{i}\left(u_{i+1}-u_{i}\right)^{2}+\frac{M}{2} \sum_{i} \dot{u}_{i}^{2},
$$

where $i$ runs over the sites of both chains, $K$ is the elastic constant of a $\sigma$ bond, and $M$ the mass of a $\mathrm{CH}$ group.

The various parameters used in the calculations are chosen so as to model cis-polyacetylene. The system can, however, be considered as a model system which is able to grasp the essentials of most (non-degenerate) conjugated polymers. Of course, the use of two polymers to describe the heterojunction is different from most real solar cell devices, which consist of a polymer-fulleride heterojunction. However, this difference is mostly of geometrical character and as far as the interactions are concerned, the above equations apply equally well to both type of heterojunctions.

Very recently, Miranda et al. developed a set of parameters in order to solve the inconformity of parameters when Coulomb interactions are included in the Hamiltonian. ${ }^{25,26}$ Thus, in this paper, we adopt the parameters developed by them: $t_{0}=2.1 \mathrm{eV}, \alpha=3.2 \mathrm{eV} / \AA$, $t_{e}=0.05 \mathrm{eV}, K=21.0$ $\mathrm{eV} / \AA^{2}, M=1349.14 \mathrm{eV} \mathrm{fs}{ }^{2} / \AA^{2}, r_{0}=1.22 \AA, U=4.1 \mathrm{eV}$, $\beta=3.4$. In this paper, we fixed the band offset $\Delta E=0.4$ $\mathrm{eV}$, which is slightly above the minimum offset required for charge exciton dissociation found experimentally ${ }^{15}$ as well as in our simulations. Changing this parameter to a larger value will not alter our qualitative conclusions.

Within the nonadiabatic molecular dynamics method, the electrons are treated quantum-mechanically and evolve 
according to the time-dependent Schrödinger equation,

$$
i \hbar|\dot{\Psi}\rangle=\hat{H}_{\text {elec }}|\Psi\rangle .
$$

The atoms, on the other hand, are considered as classical particles, governed by Newton equations, and move under the effects of nuclear and electronic contributions,

$$
M \ddot{u}_{i}=F_{i}(t)=-\nabla_{i}\left\langle\Psi\left|\hat{H}_{\text {latt }}\right| \Psi\right\rangle-\nabla_{i}\left\langle\Psi\left|\hat{H}_{\text {elec }}\right| \Psi\right\rangle .
$$

To include thermal effects, we use a canonical Langevin equation. ${ }^{27,28}$ For the fluctuation term, we choose a white stochastic signal $\zeta(t)$ with the following properties: $\langle\zeta(t)\rangle \equiv 0$ and $\left\langle\zeta(t) \zeta\left(t^{\prime}\right)\right\rangle=B \delta\left(t-t^{\prime}\right)$. A Stokes like dissipation term is also included. Therefore, we modify Eq. (13) to

$$
M \ddot{u}_{i}=-\gamma \dot{u}_{i}+\zeta(t)+F_{i}(t) .
$$

The relationship between $\zeta, \gamma$, and the temperature $T$ of the system is given by the fluctuation dissipation theorem,

$$
B=2 k_{B} T \gamma M .
$$

The wave functions that are solutions to Eq. (12) can be expressed as a series expansion of instantaneous wave functions. These wave functions are solutions to the time independent Schrödinger equation with a Hamiltonian calculated using the geometry of the lattice at that particular instant of time:

$$
\begin{gathered}
\hat{H}_{\text {elec }}\left|\phi_{j}\right\rangle=\varepsilon_{j}\left|\phi_{j}\right\rangle, \\
\Psi_{k}(t)=\sum_{j=1}^{N}\left|\phi_{j}\right\rangle C_{j, k}(t),
\end{gathered}
$$

where $C_{j, k}$ are the expansion coefficients. The occupation number for the instantaneous eigenstate $j$ is

$$
n_{j}(t)=\sum_{k} f_{k}\left|C_{j, k}(t)\right|^{2},
$$

where $f_{k}$ is a fixed (time-independent) distribution function $\left(f_{k}\right.$ being 0,1 , or 2 depend on the initial state occupation of the $k$ th level) that determines the occupation of the time-dependent wave functions, and $n_{j}(t)$ contains information concerning the redistribution of electrons among the instantaneous eigenstate. Note that the distribution function $f_{k}$ is used when the density matrix is calculated, which enters the equation of motion for the lattice. In this way we allow for nonradiative and nonadiabatic decay processes, but exclude radiative (dipole allowed) processes, which are related to a change in the symmetry of the electronic system.

The solution of the full many-body problem given in Eq. (12) is generally not possible and approximate methods are necessary. As we have mentioned above, we use a multiconfigurational time-dependent Hartree-Fock (MCTDHF) method introduced in Refs. 25 and 26 to solve this equation. Within this method, the appropriate spin symmetry of the electronic wavefunction is taken into account, thus allowing us to distinguish between singlet and triplet excited states. Equations (12) and (14), that govern the evolution of the system, may be numerically integrated using the method which was first introduced by Ono and Terai ${ }^{29}$ and have been used by Silva and co-workers ${ }^{30-32}$ and in our previous papers. ${ }^{33,34}$
In Sec. III below, we use the staggered order parameter $r_{i}(t)$ and the mean charge density $\bar{\rho}_{i}(t)$ to analyze and display the lattice and charge density evolution,

$$
\begin{gathered}
r_{i}(t)=\frac{(-1)^{i}}{4}\left[u_{i-1}(t)-2 u_{i}(t)+u_{i+1}(t)\right], \\
\bar{\rho}_{i}(t)=\frac{1}{4}\left[\rho_{i-1, i-1}(t)+2 \rho_{i, i}(t)+\rho_{i+1, i+1}(t)\right],
\end{gathered}
$$

where the charge density $\rho_{i, j}(t)=\sum_{s}\left\langle\hat{c}_{i, s}^{\dagger} \hat{c}_{j, s}\right\rangle$.

\section{RESULTS AND DISCUSSION}

\section{A. Exciton dissociation in the D-A polymer heterojunction}

As we have pointed out above, the D-A polymer heterojunction is constructed by two coupled polymer chains which are placed face to face. We set different values to the on-site energies of the two chains. Figure 1 shows the schematic diagram of the energy spectrum of the D-A polymer heterojunction, where $\Delta E$ is the band offset. The donor chain is labeled $1-150$, while the acceptor chain is labeled 151-300. Initially, there are no interactions between the two chains, and there is a singlet exciton in the donor chain while the acceptor chain is in the ground state. Then, the inter-chain interactions are smoothly turned on reaching their full strength within $75 \mathrm{fs}$. This way of introducing the inter-chain interaction mimics an exciton moving into the D-A polymer heterojunction.

Figure 2 shows the temporal evolution of the staggered order parameter $r_{i}(t)$ (top panel) and the mean charge density $\bar{\rho}_{i}(t)$ (bottom panel) with the temperature and the electric field set to zero and an inter-chain distance set to $5 \AA$. We can divide the whole exciton dissociation process into three stages. Stage 1 is from 0 to $t_{1}=60 \mathrm{fs}$. In this stage, the exciton keeps its shape and the charge density is zero all over the two chains. This is because the inter-chain interactions are still increasing and not large enough to make the exciton dissociate. Stage 2 is from $t_{1}$ to $t_{2}=250 \mathrm{fs}$. This is the time interval during which the exciton dissociation process takes

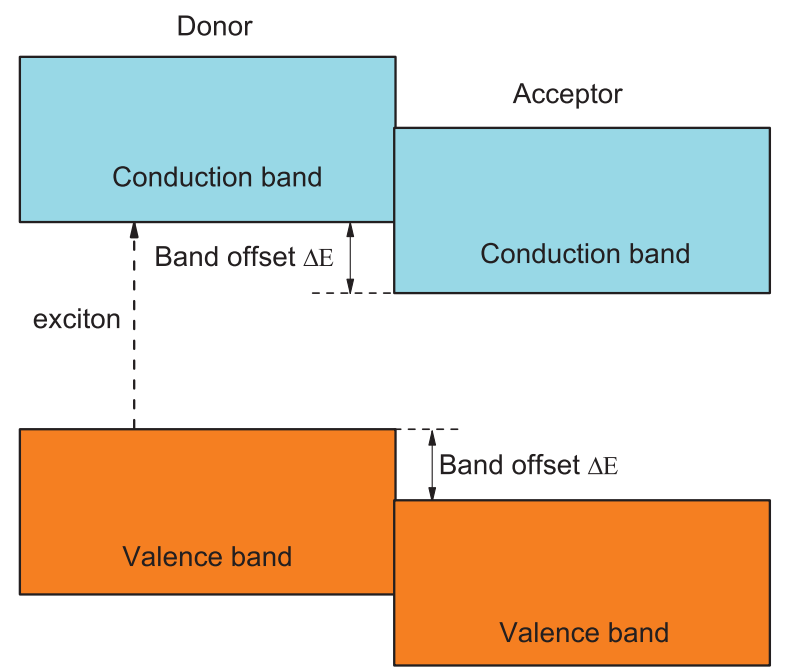

FIG. 1. Schematic diagram of the energy spectrum of a D-A polymer heterojunction. 


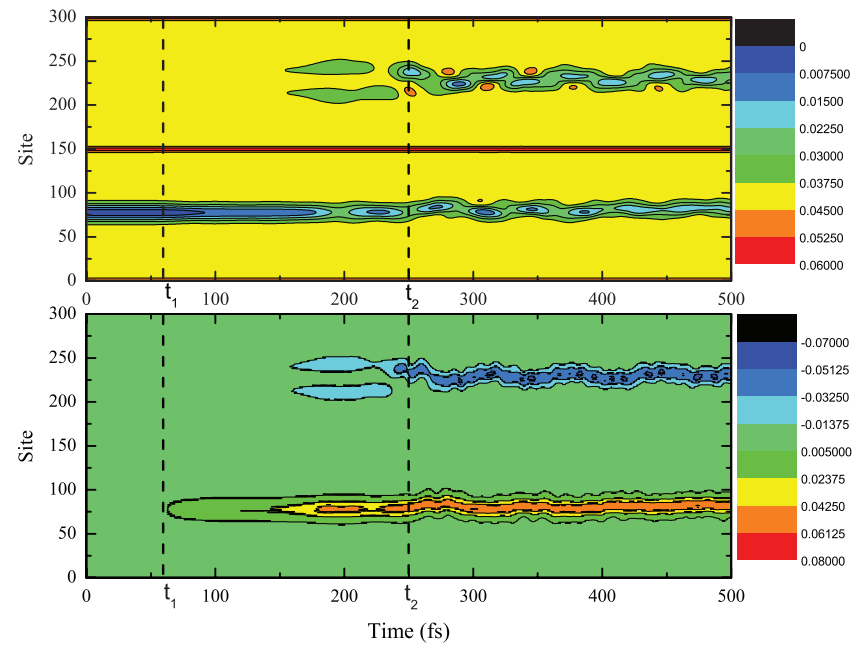

FIG. 2. Time evolution of the staggered order parameter $r_{i}(t)$ (top panel) and the mean charge density $\bar{\rho}_{i}(t)$ (bottom panel) for SE dissociation in D-A polymer heterojunction with $T=0, E=0$, and $d=5 \AA$.

place. From the top panel of Fig. 2, we see that from $t_{1}$ the exciton shape becomes more shallow and after 150 fs lattice distortions appears in the acceptor chain. From the bottom panel of Fig. 2, we can see that there are some positive charges appearing on the donor chain and some negative charges on the acceptor chain, i.e., CT state. When reaching $t_{2}$, the separation is more or less completed and integer charges with opposite sign reside on the two chains. Stage 3 is from $t_{2}$ to the end of the simulation. During this time interval we see no overall change in neither the geometrical nor the electronic structure of the system; the exciton remains completely dissociated. From the top panel, we see that the fully dissociated exciton has two lattice distortions, one on the donor chain and the other on the acceptor chain. In combination with the evolution of the mean charge density (bottom panel), we can conclude that these lattice distortions correspond to negative and positive polarons, respectively. Since the two polarons are Coulombically bound together and located on adjacent donor and acceptor chains, we refer to this CT state as a polaron pair.

In order to understand the exciton dissociation process in more detail, we now turn to the evolution of the occupation number of the instantaneous eigenstates shown in Fig. 3. The top panel of Fig. 3 displays the occupation number evolution for levels from LUMO (lowest unoccupied molecular orbital) to $\mathrm{LUMO}+8$, and the bottom panel for levels from HOMO (highest occupied molecular orbital) to HOMO-8. Note that the numbering of the levels is for the two-chain system. With Fig. 1 in mind, we can understand why the initial occupation by the excited electron is in LUMO+5. This is the lowest unoccupied level of the donor chain. In stage 1 (from 0 to $t_{1}$ ), the occupation numbers for all unoccupied levels are unchanged. At time $t_{1}$ the occupation number of LUMO+5 sharply decreases from 1 to 0 , while at the same time the occupation number of LUMO+4 sharply increases from 0 to 1 . This change in occupation number reflects the fact that these two levels interchange positions. The electron transfer associated with this change (see
Fig. 2, lower panel) is mostly due to the fact that the wavefunction corresponding to this level spreads over to the acceptor chain. In the following, we see that LUMO+4 is occupied for a very short time and then the occupied orbital further relaxes to $\mathrm{LUMO}+3$, then to $\mathrm{LUMO}+2$, and to $\mathrm{LUMO}+1$. Again, these changes do not correspond to transfer of the electron from one level to another, instead they occur as a result of changes in the eigen-energies of the respective levels.

At $t=160$ fs, we see from Fig. 2 that there are substantial changes in both the geometrical and electronic structures that occur simultaneously. These changes signal electron transfer from the donor to the acceptor chain. It is also at this time that we observe some correlated changes in both the HOMO and LUMO manifolds. The hole is rapidly moving to lower energy levels and the electron starts to spread over several LUMO levels. It is clear that the CT state is far from an eigenstate of the Hamiltonian and that both the hole and the electron reside away from the ideal situation with the hole in the HOMO level (located on the donor chain) and the electron in the LUMO level (located on the acceptor chain).

From $t_{2}$ to the end of the simulation, the electron remains distributed over several levels with some oscillatory behavior. The levels LUMO, LUMO $+5, \mathrm{LUMO}+6$, and LUMO+7 are hosting the electron and the final distribution of the hole is mainly over HOMO, HOMO-5, and HOMO-6. That is to say, the CT state is in an excited ("hot") state, which is in agreement with results from experiments..$^{20,21}$ It is interesting to note that there is no tendency for further relaxation of the electron and hole towards the band edges. This is to a large extent due to the Coulomb interactions at the D/A interface, which dramatically changes the internal field experienced by each of the two polarons as compared to an isolated polaron.

With the knowledge of these details of the dissociation process in mind, we now turn to simulations in which we have varied the inter-chain interaction strength, the temperature, and the strength of an external electric field. First, we show the results of how the exciton dissociation is affected by the inter-chain interactions with both temperature and the electric field set to zero. Figure 4 shows the net charge evolution per chain, curves (a)-(c) correspond to $d=4,5$, and $6 \AA$, respectively. Initially, the net charges of the donor and the acceptor chain are zero for three cases. Curve (b) is the same result as displayed in Fig. 3 (bottom panel) but with the charges on each chain summed up. The three stages of charge transfer discussed above are also clearly visible in this graph. By increasing the inter-chain coupling (by reducing $d$ from $5 \AA$ to $4 \AA$ ), the charge transfer process occurs much faster and is completed within about 50 fs after the full interaction strength is reached, even though there is some oscillatory behavior following this fast charge transfer process. For a lower inter-chain coupling ( $d$ set to $6 \AA$ ), the net charges of the two chains only reach about $0.2 e$, which means that the exciton does not dissociate. The state is best described as a polarization of the charge density, which occurs as a result of the difference in the on-site energy of the two chains. 

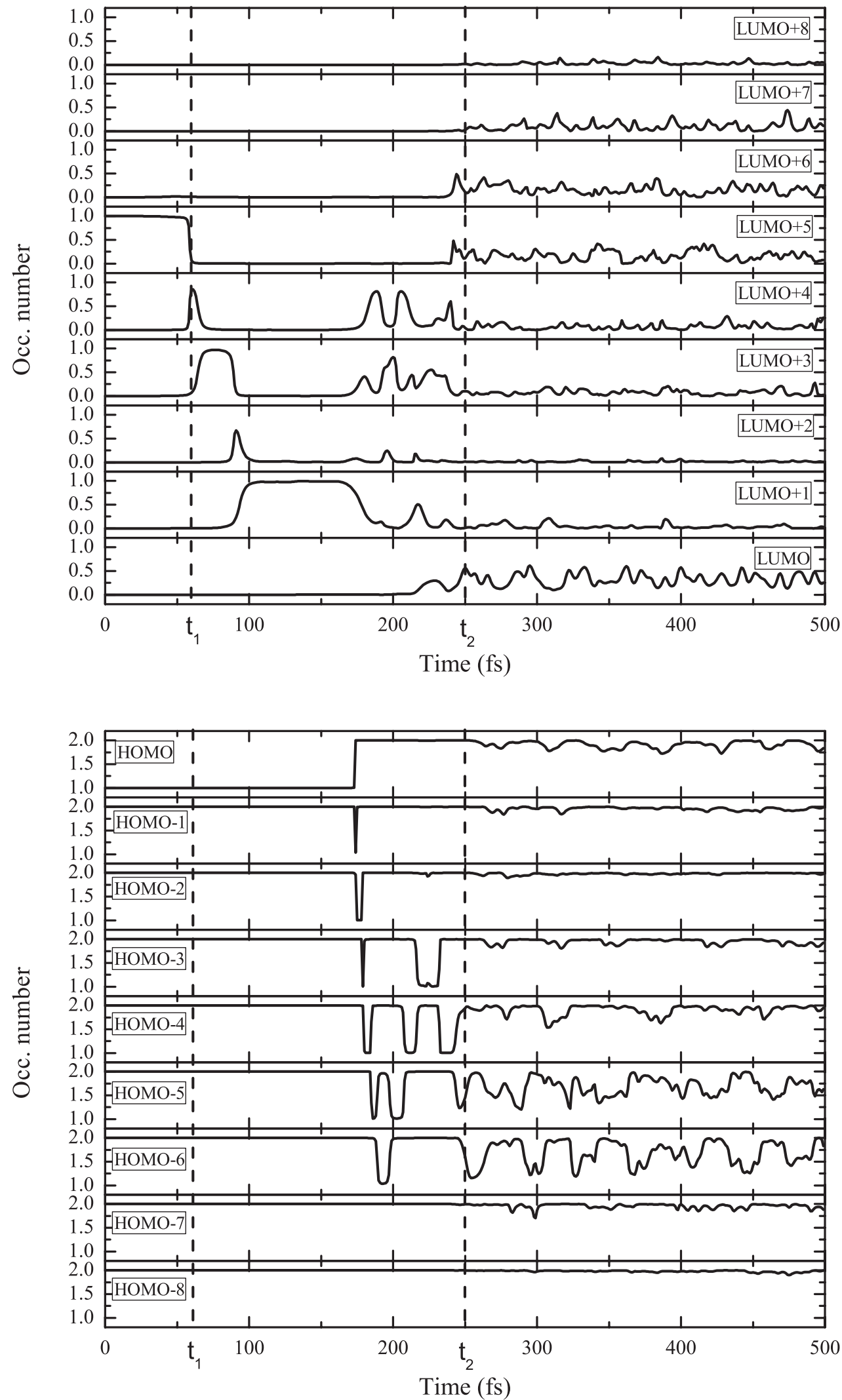

FIG. 3. Time evolution of the occupation numbers for some levels in conduction band and valence band. (From the same simulation as Fig. 2.)

To study the temperature effect on the exciton dissociation process, we again set the electric field to zero. Since we would like to point at the effect of the temperature to facilitate exciton dissociation, we chose an inter-chain distance which is large enough to result in no charge transfer at zero temperature. In the case shown in Fig. 5, this distance is set to $8 \AA$. Figure 5 shows the net charge evolution per chain, curves (a)-(c) correspond to $T=150 \mathrm{~K}, 100 \mathrm{~K}$, and $50 \mathrm{~K}$, respectively. From Fig. 4, we have seen that the exciton does not dissociate for inter-chain distance greater than $5 \AA$ in case of 


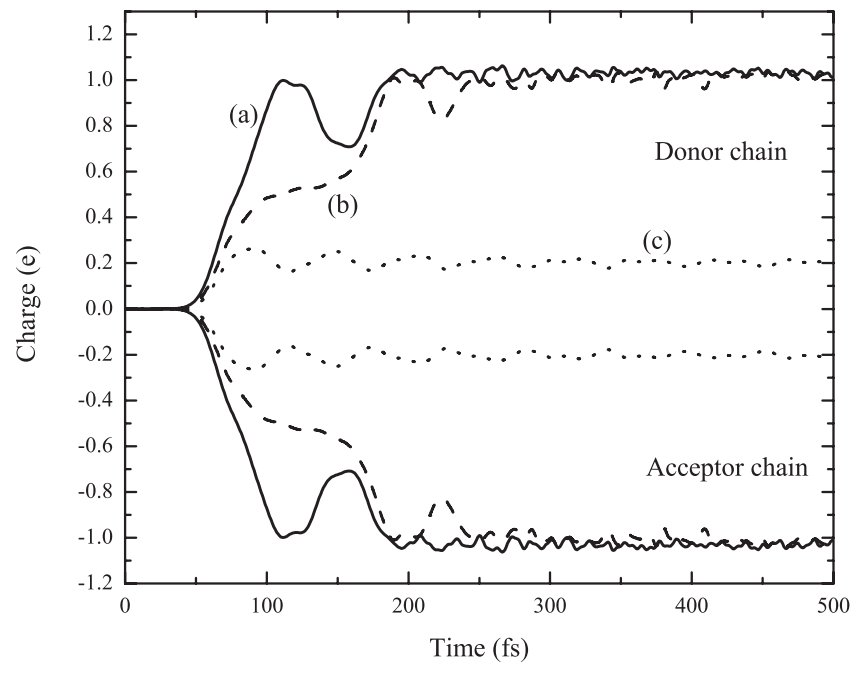

FIG. 4. Time dependence of the net charges (in units of $-|e|)$ per chain with $E=0, T=0$, and different inter-chain distance: (a) $d=4 \AA$, (b) $d=5 \AA$, and (c) $d=6 \AA$.

zero electric field and zero temperature. However, a non-zero temperature changes this result. At an inter-chain distance of $8 \AA$, we observe exciton dissociation at both $150 \mathrm{~K}$ and 100 $\mathrm{K}$ (curves (a) and (b) in Fig. 5) but not at $50 \mathrm{~K}$ (curve (c)). Obviously, exciton dissociation into a CT state is thermally stimulated.

In all the cases presented above, the polaron pair remains a stable CT state following the exciton dissociation. There is no driving force for the polaron pair to break apart into two free polaron charge carriers. The only way to overcome the Coulomb forces that bind the two oppositely charged polarons together is to introduce an external electric field directed along the chain axis of the two polymeric systems. Such a field is introduced in a set of simulations in which we again set the temperature to zero and the inter-chain distance to $8 \AA$.

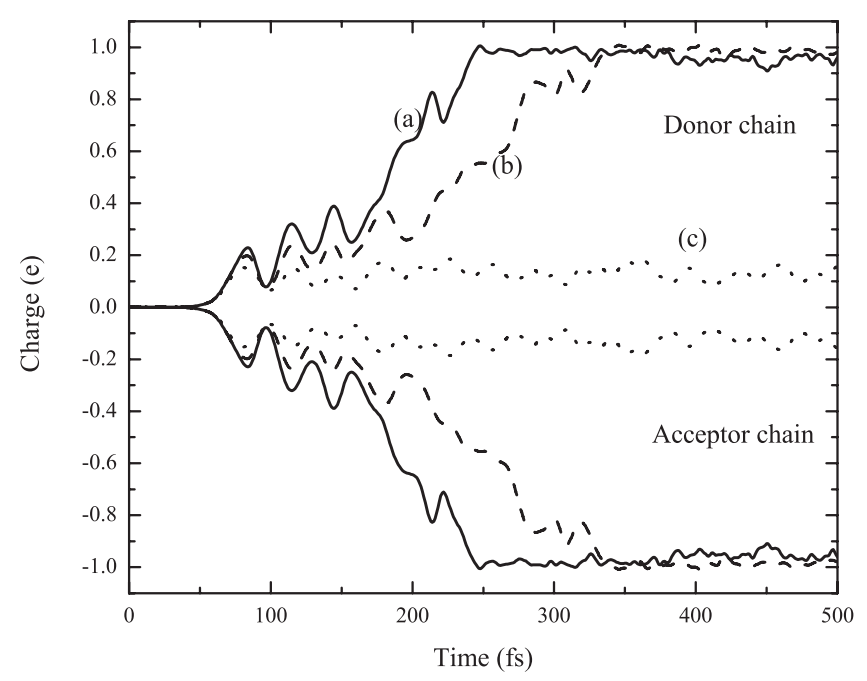

FIG. 5. Time dependence of the net charges (in units of $-|e|$ ) per chain with $E=0, d=8 \AA$, and different temperature: (a) $T=150 \mathrm{~K}$, (b) $T=100 \mathrm{~K}$, and (c) $T=50 \mathrm{~K}$.

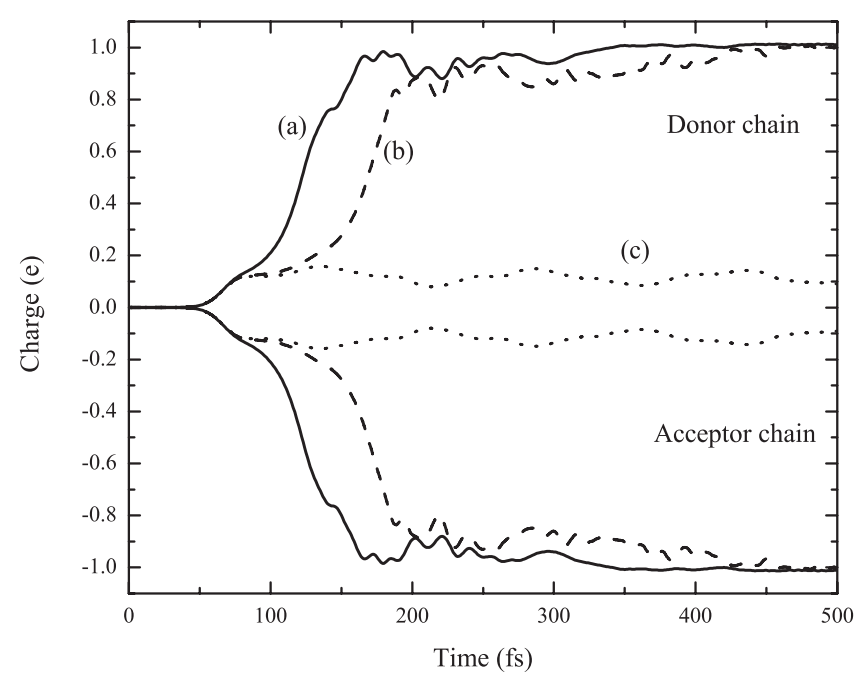

FIG. 6. Time dependence of the net charges (in units of $-|e|$ ) per chain with $T=0, d=8 \AA$, and different electric fields: (a) $E=1.4 \mathrm{mV} / \AA$, (b) $E=0.9$ $\mathrm{mV} / \AA ̊$, and (c) $E=0.8 \mathrm{mV} / \AA$.

Figure 6 shows the evolution of the net charges per chain, curves (a)-(c) correspond to $E=1.4,0.9$, and $0.8 \mathrm{mV} / \AA$, respectively. For the chosen inter-chain distance, an external electric field with strength $0.9 \mathrm{mV} / \AA$ or larger is shown to lead to exciton dissociation. From the plot in Fig. 6, the two curves (a) and (b) look very similar. However, looking at the charge density distribution for these two cases, we observe a marked difference. While case (b) looks more or less the same as the charge density distribution displayed in Fig. 2, the case with a field strength of $1.4 \mathrm{mV} / \AA$ (or larger) shows a separation of the charges into two separated polaron pairs. Thus, the electric field facilitates both exciton dissociation into a polaron pair and, at even higher field strengths, charge separation.

In Fig. 7, we display the temporal evolution of the staggered order parameter $r_{i}(t)$ (top panel) and the mean charge

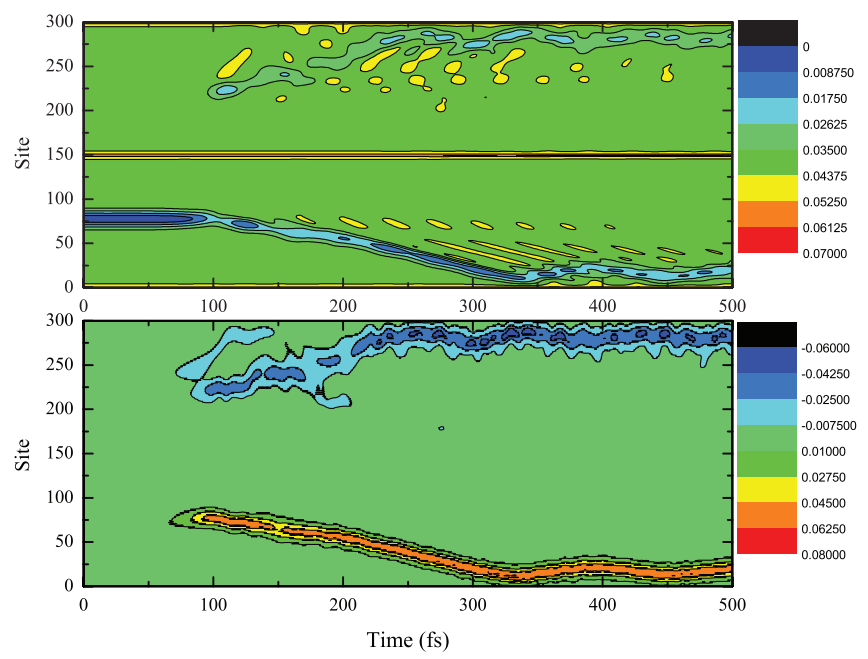

FIG. 7. Time evolution of the staggered order parameter $r_{i}(t)$ (top panel) and the mean charge density $\bar{\rho}_{i}(t)$ (bottom panel) for SE dissociation in D-A polymer heterojunction with $E=2.0 \mathrm{mV} / \AA$, $T=0$, and $d=5 \AA$. 
density $\bar{\rho}_{i}(t)$ (bottom panel) with the parameters $T=0, E$ $=2.0 \mathrm{mV} / \AA$, and $d=5 \AA$. The behavior shown in Fig. 7 displays the two steps in the photovoltaic process introduced above: exciton dissociation and charge separation. At $t=100$ fs, the exciton has dissociated into a polaron pair. In contrast to Fig. 2 (in that case, the exciton dissociates at about $250 \mathrm{fs}$ ), the exciton dissociates much faster, which again shows that the electric field contributes to the exciton dissociation. As soon as the polaron pair is created, the two oppositely charged polarons start to separate: the negative polaron moves in one direction, opposite to the direction of the electric field, and the positive polaron moves along the donor chain in the direction parallel to the electric field. Apparently, the applied field strength is enough to break the bounding effect of the Coulomb interaction between the polarons, and the polarons can be considered to be free charge carriers.

The critical electric field to separate the polaron pair at an inter-chain distance of $5 \AA$ is $2.0 \mathrm{mV} / \AA$. If the electric field is lower than this value, the two polarons will not separate. We have performed the same type of simulations at different inter-chain distances: in the case of $d=8 \AA$ presented in Fig. 6 above, the critical field for charge separation is $1.4 \mathrm{mV} / \AA$, whereas at $d=4 \AA$ the critical field is $3.3 \mathrm{mV} / \AA$. Thus, the closer the inter-chain distance is, the stronger is the binding of the polaron pair.

If we summarize the observations made above concerning the effect of different inter-chain distances, we reach the conclusion that the exciton dissociation into a polaron pair is facilitated the closer the chains are, whereas the charge separation into free polarons is more difficult at short distances. Since both processes are necessary for the conversion of the solar energy into electrical energy, there is ideally an optimal distance for which the required process occurs with highest probability. It is probably rather difficult to control the separation distance between the donor and acceptor units in a real device but nevertheless this effect should be kept in mind. If the limiting step in the device performance is found to be the charge separation process, it would be preferable, by means of structural modifications, to separate these units somewhat and study the effect of this change on the device performance.

\section{B. Exciton dissociation in the D-D-A-A polymer heterojunction}

In order to get some insight into the separation of the polaron pair into free polarons in a direction perpendicular to the heterojunction, we have introduced the D-D-A-A system. The D-D-A-A polymer heterojunction is constructed by two Dchains and two A-chains. These chains are placed face to face with equal inter-chain distances. Initially, the exciton locates on the inner D-chain. Then, we smoothly turn on all the interchain interactions and study the same processes as discussed above, namely, the exciton dissociation and the separation of the polaron pair.

In relation to what was presented above for the D-A system, we note that, by extending the system to four chains, exciton dissociation occurs more easily and at larger interchain distances than for the two-chain system. It is also clear from the simulations that it is possible to separate the polaron pair also in the direction perpendicular to the chain axis if an external electric field is applied in this direction. Since the geometrical constraints are different compared the case of polaron pair separation along the chain axis, we cannot make direct comparison with the case presented above. In particular, it is only possible to move the polaron one interchain distance away from the heterojunction, from the inner to the outer Dchain (A-chain). In this configuration, the two polarons are still Coulombically bound, much weaker though than in the initial state, and the potential drop due to the electric field is rather limited.

As an example, we find that in case of $T=0$ and $d=10$ $\AA$, the critical electric field for this kind of charge separation is $7.0 \mathrm{mV} / \AA$. The total potential drop the system experiences when two polarons move from the inner to the outer chains is thus $140 \mathrm{meV}$, i.e., even though the critical field strength is rather high, we can conclude that the polaron pair is not very strongly bound. For a system in which it would have been possible for the polarons to move further away from the heterojunction, the critical field would be considerably reduced as compared to the value observed for the D-D-A-A system.

\section{SUMMARY AND CONCLUSIONS}

We have simulated the exciton dissociation process in a D-A polymer heterojunction by solving the timedependent Schrödinger equation and the lattice equation of motion simultaneously and nonadiabatically using the SuSchrieffer-Heeger Hamiltonian with additional contributions from electron-electron interactions and an external electric field. We have also used the Langevin approach to consider the temperature effects.

The exciton, initially localized to the donor chain, is brought in contact with an acceptor chain with a LUMO (HOMO) level $0.4 \mathrm{eV}$ lower in energy as compared to the corresponding levels in the donor system. The process of electron transfer to the acceptor chain is studied in detail as a function of time. The processes that lead to charge separation include both energy level crossings and truly nonadiabatic effects such as electron transfer between levels and spreading of the occupation of the instantaneous eigenstates over several such levels. The final state corresponds to a CT state, which is a polaron pair, with the positively (negatively) charged polarons localized to the donor (acceptor) chain. The energy diagram is however different from the usual polaron electronic structure with gap states with integer occupation numbers $(0$, 1, or 2). Here, the CT state is a "hot" state with non-integer occupation numbers of several of the levels in the HOMO and LUMO bands of the system.

The effect of the inter-chain distance, the temperature, and the electric field on the charge transfer process are investigated. We found that, on the one hand, closer inter-chain distance makes it easy for the exciton to dissociate and form a polaron pair, but on the other hand makes it more difficult for the polaron pair to dissociate into state with separated non-interacting polarons. This indicates that we need a compromise in designing real solar cell device to obtain the highest possible efficiency. We also found that both the temperature and the electric field have apparent effects on the 
exciton dissociation. Higher temperature and higher electric field strength make it easier for the exciton to dissociate into the state with two Coulombically bound polarons and for this polaron pair state to dissociate into the charge separation state.

\section{ACKNOWLEDGMENTS}

The authors would like to thank Patrick Norman, Bo Durbeej and Zhong An for valuable discussions. Financial support from the Swedish Research Council and from the Swedish Energy Agency is gratefully acknowledged.

${ }^{1}$ N. S. Sariciftci, L. Smilowitz, A. L. Heeger, and F. Wudl, Science 258, 1474 (1992).

${ }^{2}$ G. Yu, J. Gao, J. C. Hummelen, F. Wudl, and A. J. Heeger, Science 270, 1789 (1995)

${ }^{3}$ S. H. Park, A. Roy, S. Beaupre, S. Cho, N. Coates, J. S. Moon, D. Moses, M. Leclerc, K. Lee, and A. J. Heeger, Nature Photon. 3, 297 (2009).

${ }^{4}$ J. Y. Kim, K. Lee, N. E. Coates, D. Moses, T. Q. Nguyen, M. Dante, and A. J. Heeger, Science 317, 222 (2007).

${ }^{5}$ T. Ameri, G. Dennler, C. Lungenschmied, and C. J. Brabec, Energy Environ. Sci. 2, 347 (2009)

${ }^{6}$ L. Dou, J. You, J. Yang, C. C. Chen, Y. He, S. Murase, T. Moriarty, K. Emery, G. Li, and Y. Yang, Nature Photon. 6, 180 (2012).

${ }^{7}$ M. A. Green, K. Emery, Y. Hishikawa, W. Warta, and E. D. Dunlop, Prog. Photovoltaics 20, 12 (2012).

${ }^{8}$ Primary Photoexcitations in Conjugated Polymers, edited by N. S. Sariciftci (World Scientific, Singapore, 1997).

${ }^{9}$ D. Hertel and H. Baessler, ChemPhysChem 9, 666 (2008).

${ }^{10}$ J.-L. Brédas, J. Cornil, and A. J. Heeger, Adv. Mater. 8, 447 (1996).

${ }^{11}$ V. I. Arkhipov and H. Bässler, Phys. Status Solidi A 201, 1152 (2004).

${ }^{12}$ G. A. Chamberlain, Sol. Cells 8, 47 (1983).
${ }^{13}$ C. Deibel and V. Dyakonov, Rep. Prog. Phys. 73, 096401 (2010).

${ }^{14}$ T. M. Clarke and J. R. Durrant, Chem. Rev. 110, 6736 (2010).

${ }^{15}$ B. C. Thompson and J. M. J. Frechet, Angew. Chem., Int. Ed. 47, 58 (2008).

${ }^{16}$ E. R. Bittner, J. G. S. Ramon, and S. Karabunarliev, J. Chem. Phys. 122, 214719 (2005).

${ }^{17}$ H. Tamura, E. R. Bittner, and I. Burghardt, J. Chem. Phys. 126, 021103 (2007).

${ }^{18}$ K. Gao, S. J. Xie, Y. Sun, and D. S. Liu, Org. Electron. 12, 1010 (2011).

${ }^{19}$ H. Y. Chen, J. Hou, S. Zhang, Y. Liang, G. Yang, Y. Yang, L. Yu, Y. Wu, and G. Li, Nature Photon. 3, 649 (2009).

${ }^{20}$ M. Muntwiler, Q. X. Yang, W. A. Tisdale, and X. Y. Zhu, Phys. Rev. Lett. 101, 196403 (2008).

${ }^{21}$ X. Y. Zhu, Q. Yang, and M. Muntwiler, Acc. Chem. Res. 42, 1779 (2009).

${ }^{22}$ J.-L. Brédas, D. Beljonne, V. Coropceanu, and J. Cornil, Chem. Rev. 104, 4971 (2004).

${ }^{23}$ J.-L. Brédas, J. E. Norton, J. Cornil, and V. Coropceanu, Acc. Chem. Res. 42, 1691 (2009).

${ }^{24}$ K. Ohno, Theor. Chim. Acta 2, 219 (1964).

${ }^{25}$ R. P. Miranda, A. J. Fisher, L. Stella, and A. P. Horsfield, J. Chem. Phys. 134, 244101 (2011).

${ }^{26}$ R. P. Miranda, A. J. Fisher, L. Stella, and A. P. Horsfield, J. Chem. Phys. 134, 244102 (2011).

${ }^{27}$ H. J. C. Berendsen, J. P. M. Postma, W. F. van Gunsteren, A. DiNola, and J. R. Haak, J. Chem. Phys. 81, 3684 (1984).

${ }^{28}$ L. A. Ribeiro, P. H. Oliveira Neto, W. F. da Cunha, L. F. Roncaratti, R. Gargano, D. A. da Silva Filho, and G. M. e Silva, J. Chem. Phys. 135, 224901 (2011).

${ }^{29}$ Y. Ono and A. Terai, J. Phys. Soc. Jpn. 59, 2893 (1990).

${ }^{30}$ G. M. e Silva, Phys. Rev. B 61, 10777 (2000).

${ }^{31}$ G. da Silva Pinheiro and G. Magela e Silva, Phys. Rev. B 65, 094304 (2002).

${ }^{32}$ M. P. Lima and G. M. e Silva, Phys. Rev. B 74, 224304 (2006).

${ }^{33}$ Z. Sun, D. S. Liu, S. Stafström, and Z. An, J. Chem. Phys. 134, 044906 (2011).

${ }^{34}$ Z. Sun and S. Stafström, J. Chem. Phys. 135, 074902 (2011). 